Revue d'histoire de l'Amérique française

MQ REVUE D.HISTOIRE DE L'AMÉRIQUE FRANÇAISE

\title{
Analyse de l'exposé de N.-E. Dionne sur le discours de Pierre Bédard au sujet de la langue officielle, 1793
}

E. Murray Greenwood

Volume 30, numéro 2, septembre 1976

URI : https://id.erudit.org/iderudit/303530ar

DOI : https://doi.org/10.7202/303530ar

Aller au sommaire du numéro

Éditeur(s)

Institut d'histoire de l'Amérique française

ISSN

0035-2357 (imprimé)

1492-1383 (numérique)

Découvrir la revue

Citer cette note

Murray Greenwood, E. (1976). Analyse de l'exposé de N.-E. Dionne sur le discours de Pierre Bédard au sujet de la langue officielle, 1793. Revue d'histoire de l'Amérique française, 30(2), 259-262. https://doi.org/10.7202/303530ar d'utilisation que vous pouvez consulter en ligne. 


\section{ANALYSE DE L'EXPOSÉ DE N.-E. DIONNE SUR LE DISCOURS DE PIERRE BÉDARD AU SUJET DE LA LANGUE OFFICIELLE, 1793.

\author{
Département d'bistoire \\ University of British Columbia
} \\ E. Murray GreenwOOD}

Dans son analyse du conflit linguistique de 1792-1793 à l'Assemblée législative du Bas-Canada, Mason Wade cite un discours de l'avocat Pierre-Stanislas Bédard, député de Northumberland et futur chef du Parti canadien ${ }^{1}$. Cependant, comme la Gazette de Québec n'a publié que les discours de Gabriel-Elzéar Taschereau, de Philippe de Rocheblave et de Michel Chartier de Lotbinière ${ }^{2}$, et que, ni Garneau ni Chapais n'ont pu retrouver un texte sur les observations de Bédard ${ }^{3}$, il m'a paru intéressant de connaître la source de Wade.

Dans une note, Wade fait référence au livre de NarcisseEutrope Dionne, Pierre Bédard et ses fils ${ }^{4}$. En effet, Dionne introduit, sans guillemets, ce qu'il prétend être des extraits d'un discours de Bédard, qui, conclut-il, «lui attira la sympathie de ses collègues et l'admiration du public». On peut difficilement mettre en doute

\footnotetext{
The French Canadians, 1760-1967, éd. rev. (2 vol., Toronto, 1968), I: 96, 147 , note 10 .

231 janv., 7 fév. 1793. D'après "A Bye Stander», (idem, 14 fév. 1793), les principaux orateurs favorisant un statut officiel pour le français furent Bédard, Joseph Papineau, Pierre-Amable De Bonne, et les trois autres déjà mentionnés dans le texte.

F.-X. Garneau, Histoire du Canada depuis sa découverte jusqu'à nos jours (4 e éd., 3 vol., Montréal, 1882), III: livre XIII, chap. 1; Thomas Chapais, Cours d'histoire du Canada (8 vol., Québec, 1919-1934), II: 55-82.

4 (Québec, 1909), 21-23.
} 
cette conclusion puisque le supposé disccurs de Bédard, contrairement à certains autres ${ }^{5}$, était empreint d'une note de défi:

Si le conquis doit parler la langue du conquérant, pourquoi les Anglais ne parlent-ils plus le normand?... Les Canadiens sont des conquis, mais des conquis qui sauront montrer aux autres nations le chemin de l'honneur... Ceux qui parlent d'obliger à parler une langue plutôt qu'une autre... ne savent pas ce qu'ils veulent dire, à moins qu'ils n'entendent qu'il faut exterminer tous ceux qui ne comprennent ni ne parlent cette langue.

Toutefois, on ne saurait accepter le compte rendu de Dionne. Tout semble indiquer qu'une de ses sources provenait d'un commentaire publié dans le journal Le Canadien du 30 déc. 1809, sur les vues francophobes du voyageur anglais Hugh Gray ${ }^{6}$. Le texte de Dionne et le commentaire en question sont pratiquement identiques. On y trouve, par exemple, le passage suivant: "Ceux qui parlent d'obliger de parler une langue dans un pays, ne savent pas ce qu'ils veulent dire, à moins qu'ils n'entendent qu'il faut exterminer tous ceux qui n'entendent pas et ne parlent pas cette langue. " L'exemple suivant est également frappant:

5 De Lotbinière et de Rocheblave, par exemple, insistèrent sur les problèmes pratiques de la question et proposèrent de faire preuve de patience jusqu'à ce que l'éducation du public soit parvenue à un point où l'anglais pourrait devenir la seule langue officielle. Néanmoins, il faut considérer avec réserve l'hypothèse de Michel Brunet suivant laquelle les seigneurs ayant pris la parole en Chambre ont adopté un ton d'excuse [La présence anglaise et les Canadiens (Montréal, 1964), 106-109]. Il est vrai que Taschereau a décrit l'anglais comme une langue "que nous n'avons pas le bonheur d'entendre et de parler dans ce moment", mais sa conclusion n'avait rien d'apologétique. Si, dit-il, on en venait à imposer une seule langue officielle, "notre liberté devenant une illusion prouveroit notre esclavage». De plus, il semble que Pierre-Amable De Bonne - alors dans ses jours pré-chouayens - ait adopté une attitude agressive. John Painter, marchand de Québec, a décrit la position de De Bonne comme suit: "...said that the Constitution was given for the express benefit of the Canadians, without paying any respect to the few old subjects inhabiting here, $\&$ that if these latter did not like the mode which the Canadians might think necessary to adopt in enacting laws, they might easily retire from the Country by the same road they enterd [sic] it-», Painter à James Morrison, 24 janv. 1793, Papiers Lindsay-Morrison, vol. 1: 361-363, Archives Publiques du Canada (APC'). Je remercie Pierre Tousignant de m'avoir indiqué certaines nuances dans le discours de Taschereau.

6 Letters from Canada (London, 1809). 
DIONNE

La langue anglaise est introduite dans la province de Québec [sic] autant qu'elle peut l'être. Les hommes instruits la parlent généralement, parce que le texte de la loi est anglais; la langue dans laquelle s'exprime le représentant du roi est anglaise. La pensée d'introduire la langue des vainqueurs dans les cours de justice plus qu'elle ne l'est, serait absurde aux yeux de ceux qui ont une juste idée de ce qui s'y pratique.
Le Canadien, 30 déc. 1809

La langue angloise est autant introduite dans le pays qu'il est possible qu'elle le soit. La langue de la législature est angloise, parce que le texte de la loi est Anglois et que la langue dans laquelle s'exprime le représentant du Roi est angloise, - la pensée d'introduire cette langue plus qu'elle n'est dans les Cours de Justice est absurde pour tous ceux qui ont une idée de la chose.

Il est peu probable que cet extrait du journal Le Canadien et le compte rendu de Dionne proviennent d'un troisième document rapportant ce que Bédard aurait dit devant l'Assemblée législative en 1793. Un des passages communs est que «le texte de la loi est anglais/Anglois». Notons que cette question capitale lors des discussions sur la langue ${ }^{7}$ n'a été réglée qu'après la session lorsque le secrétaire d'État, Henry Dundas, donna instruction au gouverneur Dorchester de permettre la traduction française des procédures législatives mais de n'autoriser comme officielle que la version anglaise des projets de loi $^{8}$. Il semble donc que Dionne ait fait dire à son héros des paroles qui ne furent jamais prononcées en Chambre.

Le discours passionné attribué à Bédard en 1793 a probablement contribué à l'interprétation erronée suivant laquelle il devint le chef des députés canadiens presque dès le début de la nouvelle cons-

7 Le débat s'est engagé à la suite d'un amendement présenté par le marchand John Richardson (21 janv.), sur la motion de De Bonne proposant que les journaux soient conservés dans les deux langues. Richardson suggéra que, bien que les journaux, projets de loi et statuts puissent être disponibles en français, la version anglaise constituerait en fait le texte officiel, l'anglais étant la langue officielle du Royaume. C'était le seul facteur important aux yeux de Richardson (John Porteous, 19 janv. 1793), Lettres de Richardson: 55, APC.

8 Dundas à Dorchester, 2 oct. 1793, CO 42, vol. 96: 213, APC. 
titution ${ }^{9}$. D'après une analyse des journaux de l'Assemblée législative au cours de la décennie qui suivit son établissement, les Canadiens les plus enclins à montrer leur opposition au gouverneur furent le notaire-arpenteur Joseph Papineau, l'avocat Jean-Antoine Panet et dans une moindre mesure, Philippe de Rocheblave. Ce sont eux, et non Bédard, qui ont été les chefs de file dans le débat des questions controversées telles que: essayer en vain d'établir un système judiciaire indépendant (1794), empêcher la suspension de l'habeas corpus $(1794,1797)$, supprimer les exactions illégales des seigneurs (1795) et faire échec au projet de loi sur les routes (1796). Comme l'a mentionné Helen Taft Manning, ce n'est qu'au moment de la semi-retraite de Papineau de la scène politique en 1800 que Bédard assuma son rôle de chef à l'Assemblée législative ${ }^{10}$.

9 Voir par exemple, Dionne, Pierre Bédard, 25-27; Francis-J. Audet, «L'honorable Pierre-Stanislas Bédard», Société Royale du Canada, Proceedings and Transactions (1936), I: 36; Francis-J. Audet et Edouard.Fabre Surveyer, Les députés au premier parlement da Bas-Canada (Montréal, 1946;, 28; Mason Wade, The French Cancadians, I: 96.

10 The Revolt of French Canada, 1800-1835 (Toronto, 1962), 67. 